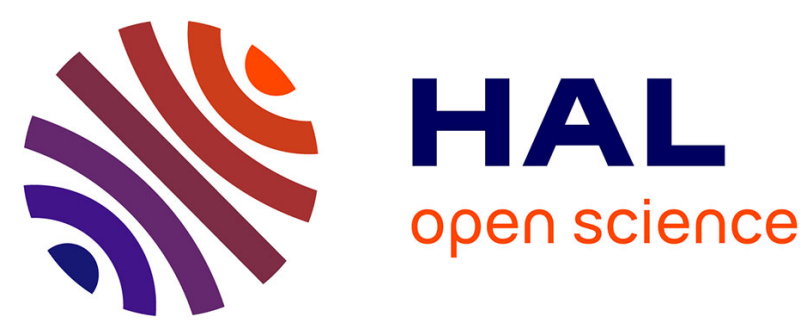

\title{
Implicit Planar and In-Plane Deformable Mapping in Medical Images Through High Order Graphs
}

\author{
Enzo Ferrante, Vivien Fecamp, Nikos Paragios
}

\section{To cite this version:}

Enzo Ferrante, Vivien Fecamp, Nikos Paragios. Implicit Planar and In-Plane Deformable Mapping in Medical Images Through High Order Graphs. IEEE International Symposium on BIOMEDICAL IMAGING: From Nano to Macro (ISBI), Apr 2015, Ney York, United States. hal-01130724

\section{HAL Id: hal-01130724 \\ https://inria.hal.science/hal-01130724}

Submitted on 12 Mar 2015

HAL is a multi-disciplinary open access archive for the deposit and dissemination of scientific research documents, whether they are published or not. The documents may come from teaching and research institutions in France or abroad, or from public or private research centers.
L'archive ouverte pluridisciplinaire HAL, est destinée au dépôt et à la diffusion de documents scientifiques de niveau recherche, publiés ou non, émanant des établissements d'enseignement et de recherche français ou étrangers, des laboratoires publics ou privés. 


\title{
IMPLICIT PLANAR AND IN-PLANE DEFORMABLE MAPPING IN MEDICAL IMAGES THROUGH HIGH ORDER GRAPHS
}

\author{
Enzo Ferrante, Vivien Fecamp, Nikos Paragios \\ GALEN Team (INRIA), Center for Visual Computing (Ecole Centrale Paris) \\ Grande Voie des Vignes 92295 Chatenay-Malabry, France
}

\begin{abstract}
In this paper we propose a novel method based on discrete optimization of high order graphs, to perform deformable sliceto-volume registration of 2D images and 3D volumes. To this end, a 2D grid superimposed to the image is considered with control points deforming in 3D and their deformations corresponding to the label space. Geometrical consistency (unique plane selection) and deformation smoothness (in-plane deformations) as well as image similarity (visual matching) are encoded in different third order cliques. The proposed formulation is optimized through its mapping to a factor graph using conventional graph optimization methods. A dataset composed of 2D slices and 3D MRI volumes of the heart was used to evaluate its accuracy leading to very promising results.
\end{abstract}

Index Terms - Slice-to-volume registration, high order graphs

\section{INTRODUCTION}

Slice-to-volume (2D to $3 \mathrm{D})$ registration has shown to be useful in different medical applications such as radio frequency liver ablation, image guided surgeries (IGS) and image guided biopsies. A variety of methods based on different techniques has been proposed in the past to deal with this challenging problem, including phase correlation [1], variational approaches [2], simulated annealing [3] and point correspondences [4]. Other approaches as graph based techniques, have been widely used to solve the problem of non-rigid image registration in the last years [5], but most of these works focus on $2 \mathrm{D}-2 \mathrm{D}$ or $3 \mathrm{D}-3 \mathrm{D}$ registration. The closest work to our proposal is [6]. In this work, authors proposed a novel formulation based on discrete optimization of Markov Random Fields (MRF) that infers the optimal labeling of a pairwise (MRF) over a 5-dimensional label space. Despite the theoretical promises of this approach, the over-parameterized label space becomes a concern in inference since the computational burden is significant. Furthermore, the plane selection is explicitly modeled through local terms which, even if propagated, cannot guarantee uniqueness of the solution. In this

Research partially supported by European Research Council Starting Grant Diocles (ERC-STG-259112) paper we propose a novel method for 2D-to-3D registration through the $3 \mathrm{D}$ deformation of a $2 \mathrm{D}$ plane. To this end we adopt a third-order potential graphical model. Triplets of control points (forming a line) are considered to encode the planarity as well as the in-plane smoothness deformation constraints. Data terms are encoded in triangle triplets providing an exact estimation of the similarity criterion between the $2 \mathrm{D}$ image and the corresponding 3D volume. Last, but not least, global consistency is imposed through the membership of the same control points into multiple third order geometric cliques. The resulting formulation is scalable and modular with respect to the similarity transformation as well as the planar deformation.

\section{NON-RIGID SLICE-TO-VOLUME REGISTRATION THROUGH HIGH ORDER GRAPHS}

Given a 2D source image $I$ and a 3D target volume $J$, we seek the slice from the volume $J$ (namely $\hat{\pi}[J]$ ) that best matches the image $I$. Image $I$ can suffer in-plane deformations modeled by a $2 \mathrm{D}$ deformation field $\hat{T}_{D}$ that is inferred during the optimization process. Even if it is possible to also consider out-of-plane deformations, in this work we just model in-plane deformations, because of the applications we are targeting (mainly IGS where the main interest consists in showing the deformed 2D image together with the corresponding slice $\hat{\pi}[J]$ ).

Our discrete formulation of the 2D-3D non-rigid registration problem consists of an undirected graph $G=<V, E>$ (a regular lattice) superimposed to the $2 \mathrm{D}$ image domain with a set of nodes $V$ and a set of third-order cliques $E=E_{D} \cup E_{R}$. The nodes are interpreted as control points of a bidimensional quasi-planar grid that models at the same time the in-plane deformations and the current position of the 2D image into the 3D volume. In order to represent those deformations, the grid is interpreted as a Free Form Deformation model (FFD) where each control point has only local influence on the deformation. Vertices in $V$ are labeled with three dimensional vectors $l_{i} \in L$ (where $L$ corresponds to a 3-dimensional label space formed by 3D displacement vectors), indicating the position of the control point in the space. Regarding the cliques in $E$, we define two different types. Cliques in $E_{D}$ are triplets 
of vertices with a triangular shape (Figure 1.a) and they are associated with data terms. Those in $E_{R}$, are horizontal and vertical collinear third-order cliques (Figure 1.b) associated with regularization terms. The control points (vertices) are displaced by assigning them different labels until an optimal position is found. In order to reach such an optimal position, let us now define the corresponding high order objective function.

The energy (objective function) to be minimized consists of data terms $D_{i j k}$ associated with triangular triplets of graph vertices $(i, j, k) \in E_{D}$ and regularization terms $R_{i j k}$ associated with collinear horizontal and vertical triplets $(i, j, k) \in$ $E_{R}$. The first ones encode the matching cost, whereas the later ones act as regularizers of the planar and regular grid structure. The minimization energy problem in the context of high order graphs is thus defined as:

$\min \sum_{(i, j, k) \in E_{D}} D_{i j k}\left(\boldsymbol{l}_{\boldsymbol{i}}, \boldsymbol{l}_{\boldsymbol{j}}, \boldsymbol{l}_{\boldsymbol{k}}\right)+\beta \sum_{(i, j, k) \in E_{R}} R_{i j k}\left(\boldsymbol{l}_{\boldsymbol{i}}, \boldsymbol{l}_{\boldsymbol{j}}, \boldsymbol{l}_{\boldsymbol{k}}\right)$,

where $l_{\boldsymbol{i}}$ is a label associated to a displacement vector $\left(d_{x}, d_{y}, d_{z}\right)$ and assigned to the node $i$, and $\beta$ is a parameter weighting the contribution of the geometric and similarity terms.

Data Term. It is defined over a set of triangular cliques as indicates Figure 1.a. Its formulation is independent of the similarity measure $\delta$ and it is calculated for each clique $\boldsymbol{d}=(i, j, k) \in E_{D}$ using the source 2D image $I$ and the corresponding plane $\pi_{d}[J]$ extracted from the target volume $J$, defined by the three control points of the clique. For a given similarity measure $\delta$, the data term associated with the clique $\boldsymbol{d}$ is thus defined as:

$$
D_{i j k}\left(\boldsymbol{l}_{\boldsymbol{i}}, \boldsymbol{l}_{\boldsymbol{j}}, \boldsymbol{l}_{\boldsymbol{k}}\right)=\int_{\Omega_{d}} \delta\left(I(\boldsymbol{x}), \pi_{d}[J](\boldsymbol{x})\right) d \boldsymbol{x},
$$

where $\boldsymbol{x} \in \Omega_{d}$, and $\Omega_{d}$ corresponds to the triangular area defined by the control points of clique $\boldsymbol{d}$ over the plane $\pi_{d}[J]$, after applying the corresponding labels $\boldsymbol{l}_{\boldsymbol{i}}, \boldsymbol{l}_{j}, \boldsymbol{l}_{\boldsymbol{k}}$ to the vertices. For example, in monomodal scenarios, a simple but efficient similarity measure is the Sum of Absolute Differences of the intensities in the patch. Since our approach is independent of the similarity measure, it could be adapted to different scenarios just by choosing an appropriate $\delta$.

Regularization Term. It imposes the planar and regular structure of the grid. We define a clique for every set of three collinear and contiguous grid nodes (in horizontal and vertical directions as depicts Figure 1.b) and associate regularization terms $R_{i j k}$ with them. We also introduce extra collinear cliques formed by nodes that are collinear but not contiguous. The aim is to propagate further the regularization so that the planar structure is conserved. The regularization term is formed by two parts: the first one, cares about the

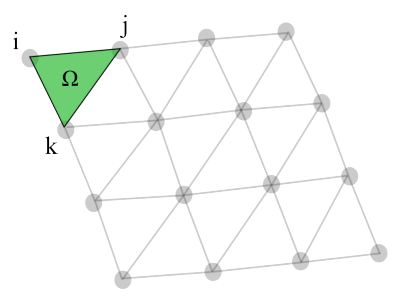

(a)

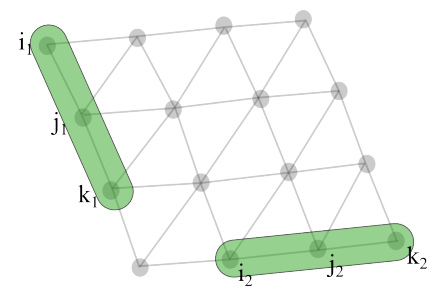

(b)
Fig. 1. (a) The green patch $\Omega$ is one of the triangular cliques $(i, j, k) \in E_{D}$ and it is used to calculate the data term. (b) Vertical $\left(i_{1}, j_{1}, k_{1}\right) \in E_{R}$ and horizontal $\left(i_{2}, j_{2}, k_{2}\right) \in E_{R}$ collinear third-order cliques used to regularize the grid.

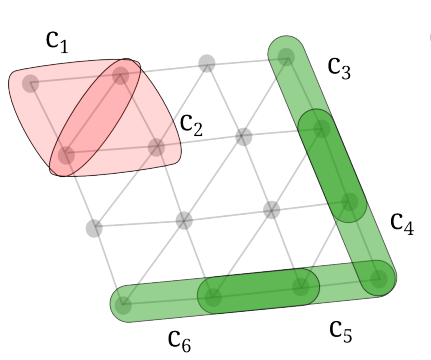

(a)

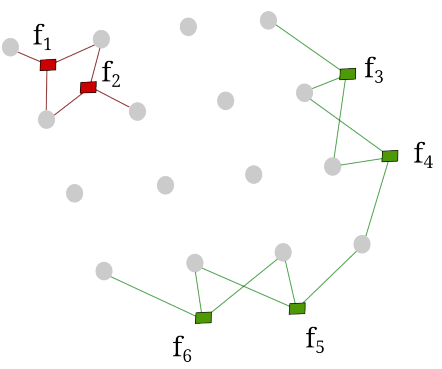

(b)
Fig. 2. Equivalence between cliques $c_{i}$ in a regular high order graph (Figure (a)) and factors $f_{i}$ in a factor graph (Figure (b)). In red, we can observe some triangular cliques (factors) associated to data terms, while in green are represented the collinear cliques (factors) associated to regularization terms.

plane structure of the grid, while the second one regularizes the in-plane deformations.

Planar Consistency can be easily enforced through a discrete approximation of the second-order derivatives of the grid. [7] points out that triplets of collinear points allows us to encode a smoothness prior based on the discrete approximation of the second-order derivatives using only the vertices position. This term checks collinearity among the three points, based on the discrete approximation of the secondorder derivatives. Thus, it penalizes the chosen labels when collinearity is not preserved. Given three contiguous control points $\left(\boldsymbol{p}_{\boldsymbol{i}}, \boldsymbol{p}_{\boldsymbol{j}}, \boldsymbol{p}_{\boldsymbol{k}}\right)$ and their corresponding displacement labels $\left(\boldsymbol{l}_{\boldsymbol{i}}, \boldsymbol{l}_{\boldsymbol{j}}, \boldsymbol{l}_{\boldsymbol{k}}\right)$ the collinearity condition is fulfilled when $\left(\boldsymbol{p}_{\boldsymbol{i}}+\boldsymbol{l}_{\boldsymbol{i}}\right)+\left(\boldsymbol{p}_{\boldsymbol{k}}+\boldsymbol{l}_{\boldsymbol{k}}\right)-2 *\left(\boldsymbol{p}_{\boldsymbol{j}}+\boldsymbol{l}_{\boldsymbol{j}}\right)=\overrightarrow{0}$.

Based on this idea, we define the following energy term using the euclidean norm of the resulting vector, normalized with the distance between the control points $d$ :

$R_{i j k}^{A}\left(\boldsymbol{l}_{\boldsymbol{i}}, \boldsymbol{l}_{\boldsymbol{j}}, \boldsymbol{l}_{\boldsymbol{k}}\right)=\frac{\left\|\left(\boldsymbol{p}_{\boldsymbol{i}}+\boldsymbol{l}_{\boldsymbol{i}}\right)+\left(\boldsymbol{p}_{\boldsymbol{k}}+\boldsymbol{l}_{\boldsymbol{k}}\right)-2 *\left(\boldsymbol{p}_{\boldsymbol{j}}+\boldsymbol{l}_{\boldsymbol{j}}\right)\right\|^{2}}{d^{2}}$ 
In-plane Deformation Smoothness can be achieved through a distance preserving approach. The in-plane grid deformation is controlled preserving the original distances between the control points of the grid. Since this metric is based on the euclidean distance between the points, it assumes that they are coplanar. Let us define $\psi_{i j}$ as the ratio of the euclidean distance between the displaced points $\boldsymbol{p}_{\boldsymbol{i}}+\boldsymbol{l}_{\boldsymbol{i}}$ and $\boldsymbol{p}_{\boldsymbol{j}}+\boldsymbol{l}_{\boldsymbol{j}}$ and the original distance between their initial positions $\boldsymbol{p}_{o, i}$ and $p_{o, j}$ as indicates the following equation:

$$
\psi_{i j}=\frac{\left\|\left(\boldsymbol{p}_{\boldsymbol{i}}+\boldsymbol{l}_{\boldsymbol{i}}\right)-\left(\boldsymbol{p}_{\boldsymbol{j}}+\boldsymbol{l}_{\boldsymbol{j}}\right)\right\|}{\left\|\left(\boldsymbol{p}_{\boldsymbol{o}, \boldsymbol{i}}\right)-\left(\boldsymbol{p}_{\boldsymbol{o}, \boldsymbol{j}}\right)\right\|} .
$$

Once defined $\psi_{i j}$, we need our regularizer to fulfill two conditions: first, we need it to be symmetric with respect to the displacement of the points, i.e. to penalize with the same cost when the control points are closer or more distant. This is achieved if the following condition is fulfilled $\Psi_{i j}=(1-$ $\left.\psi_{i j}\right)^{2}+\left(1-\psi_{i j}^{-1}\right)^{2}=0$. Second, we need the energy to be zero when the points are preserving distances and bigger than zero otherwise. The following expression fulfills both conditions for a couple of control points $\boldsymbol{p}_{\boldsymbol{i}}, \boldsymbol{p}_{\boldsymbol{j}}$ : So, for a given clique $(i, j, k)$ we define the second part of the regularizer as:

$$
R_{i j k}^{B}\left(\boldsymbol{l}_{\boldsymbol{i}}, \boldsymbol{l}_{\boldsymbol{j}}, \boldsymbol{l}_{\boldsymbol{k}}\right)=\frac{\Psi_{i j}+\Psi_{j k}}{2}
$$

The equation that regularizes our grid is a combination of both parts:

$$
R_{i j k}\left(\boldsymbol{l}_{\boldsymbol{i}}, \boldsymbol{l}_{\boldsymbol{j}}, \boldsymbol{l}_{\boldsymbol{k}}\right)=(1-\alpha) R_{i j k}^{A}\left(\boldsymbol{l}_{\boldsymbol{i}}, \boldsymbol{l}_{\boldsymbol{j}}, \boldsymbol{l}_{\boldsymbol{k}}\right)+\alpha R_{i j k}^{B}\left(\boldsymbol{l}_{\boldsymbol{i}}, \boldsymbol{l}_{\boldsymbol{j}}, \boldsymbol{l}_{\boldsymbol{k}}\right)
$$

where $\alpha$ is a weighting factor. The proposed objective function can be either optimized through its mapping to a factor graph, its mapping to a pairwise graph and the use of conventional optimization methods, or using higher order optimization methods like dual decomposition. In order to proceed with a proof of concept for the method, we have adopted the factor graph approach and implemented it using OpenGM2 library [8].

We derive the factor graph $G^{\prime}=<V^{\prime}, F^{\prime}, E^{\prime}>$ from the aforementioned higher order graph $G$ (see Figure 2). A factor graph is a bipartite graph that factorizes a given global energy function, expressing which variables are arguments of which local functions. $V^{\prime}$ is the set of variable nodes formed by the nodes of $G$. $F^{\prime}$ is a the set of all the factors $f \in F^{\prime}$, where every $f$ is associated to one clique from the high order graph. The set $E^{\prime} \subset V^{\prime} \times F^{\prime}$ defines the relation between the nodes and the factors. We use the previous 3-dimensional label space $L$ for labeling the graph nodes. Every factor $f$ has a function $\varphi_{f}: V^{\prime 3} \rightarrow \mathbb{R}$ associated that can correspond to a data term or regularization term from equations 2 or 6 respectively. The energy function of our discrete labeling problem in the context of factor graphs is then given by:

$$
\mathcal{E}(x)=\sum_{f \in F^{\prime}} \varphi_{f}\left(v_{i}, v_{j}, v_{k}\right),
$$
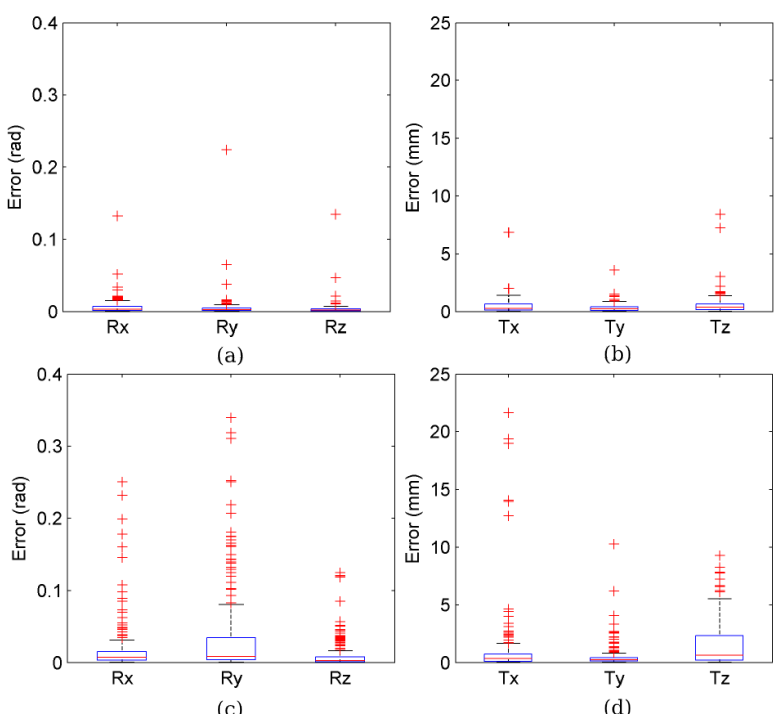

Fig. 3. Error estimation of the plane parameters for the method presented in this paper ((a) and (b)) and the pairwise MRF based approach presented in [6] ((c) and (d)) for the same dataset with equivalent setup.

where $x$ corresponds to a given labeling for the complete graph and $v_{i}, v_{j}, v_{k}$ are the variables in the neighborhood (or scope) of the factor $f$. We optimize this model using Loopy Belief Propagation algorithm implemented in OpenGM2, which can deal with third-order factors.

\section{IMPLEMENTATION \& VALIDATION}

Despite the reasonable dimension of the label space (3D), the use of factor graphs limits the number of labels that can be used during inference. In order to overcome this limitation we adopt a pyramidal approach where grid size is decremented in every pyramid level. For each grid resolution, some iterations of the registration algorithm are performed. We choose the optimal set for each one and update the control point positions while varying the size of the displacement vectors that form the label space to improve the search space sampling.

It should be noted that the planarity constraint is imposed in a soft manner. Furthermore, due to the incremental approach, errors can be accumulated and lead to inconsistent planar transformations. Therefore, regularization terms do not guarantee that the final solution is a plane. In order for the grid to be a plane, we project every control point to the regression plane estimated from the current position of these points. This projection corresponds to a 2D FFD that gives a good approximation of the deformation field $\hat{T}_{D}$.

Evaluation and comparison of the method was performed over the 2D/3D monomodal MRI heart dataset presented in [6]. It consists of 10 different temporal series of 20 bidimensional slices each one, that must be registered with a volu- 
metric MRI, giving a total of 200 registration cases. For a complete description of the dataset refer to [6]. Plan estimation was evaluated measuring the error between the ground truth and the estimated planes (planes were represented by a 6-DOF rigid transformation, with 3 translation and 3 rotation parameters $\left.\left(R_{x}, R_{y}, R_{z}, T_{x}, T_{y}, T_{z}\right)\right)$.

The average error among all the registration cases is less than $0.0057 \mathrm{rad}\left(0.3265^{\circ}\right)$ for rotation and less than $0.5386 \mathrm{~mm}$ for translation parameters (see Figure 3). Given that image resolution in $z$ axis is lower than in $x$ and $y$, a bigger error is observed in the estimated translation for $z$ coordinate. Experiments were performed using 3 grid levels, with 5 iterations each one, initial grid size of $40 \mathrm{~mm}$, maximum displacements of $25 \mathrm{~mm}, 19$ labels, $\alpha=0.5$ and $\beta=0.7$. The average running time was around 80 seconds (on an Intel Xeon W3670 with 6 Cores and 16GB of RAM), where $57 \%$ of it corresponds to energy computation and 33\% to optimization. Figure 3 compares the results of our method with the results obtained using the algorithm proposed in [6]. Both mean and standard deviation errors of the estimated parameters are reduced by our method, meaning that the results are more accurate and less disperse than in the previous case. It shows that improving the quality of the energy terms by increasing the order of the cliques, results in an improvement of the final solution. It is important to remark that, for the same number of grid levels and iterations, our new approach reported slightly better computational time, running on the same computer (average of $80 \mathrm{sec}$ per registration case for our method and $100 \mathrm{sec}$ for the previous method). This difference is mainly due to the over-parameterized label space (5-dimensional) adopted by the previous method, that exponentially increments the number of labels needed to sample an equivalent solution space.

Validation of in-plane deformation was performed over 20 registration cases. The dataset provides manual segmentations $\mathcal{S}$ of the left endocardium from a set of 20 slices. We register each slice with a starting from a random position around the ground truth (using gaussian noise with $\sigma_{r}=4.5^{\circ}$ and $\sigma_{t}=5 \mathrm{~mm}$ for translation and rotation parameters respectively). The estimated deformation field $T_{D_{i}}$ was applied to the corresponding initial segmentation $s_{i} \in \mathcal{S}$ and it was compared with the ground truth using DICE coefficient. The average DICE before deformation was 0.85 while after deformation we obtained 0.91 , showing that in case of deformable organs like heart, deformation is important to guarantee reliable results.

\section{CONCLUSIONS}

In this paper we proposed a novel slice-to-volume deformable registration method based on discrete optimization of higher order graphs. The method is independent of the similarity measure; consequently, it hast the potential to be adapted to different scenarios with variety of image modalities. Feature selection for different modalities is a complex topic that is out of the scope of this paper. We compared our results with another method based on graph optimization, that uses lower dimensional cliques but higher dimensional label space. We showed that using higher order cliques we can model more powerful and accurate energies that lead to more reliable results.

Future work must be conducted mainly in three directions. First, the validation of the proposed formulation in real clinical scenarios (liver tumor resection guidance and MRI/US guidance for prostate biopsy) is under investigation. It should be noted that such a task is complex due to the complete absences of ground truth. Second, alternative optimization methods (in particular the ones acting directly on the objective function label space like dual decomposition) might lead to better results and are under investigation. Last, decoupling rigid and deformable parameters would allow us to have a better control over the algorithm behavior, while also decreasing the computational complexity and reducing the hardness of the associated graphical model.

\section{REFERENCES}

[1] R. San José Estépar, C.F. Westin, and K.G. Vosburgh, "Towards real time $2 \mathrm{~d}$ to $3 \mathrm{~d}$ registration for ultrasoundguided endoscopic and laparoscopic procedures," IJCARS, vol. 4, no. 6, pp. 549-560, 2009.

[2] S Heldmann and N Papenberg, "A variational approach for volume-to-slice registration," in Scale Space and Variational Methods in Computer Vision, pp. 624-635. 2009.

[3] A Cifor, L Risser, MP Heinrich, D Chung, and JA Schnabel, "Rigid registration of untracked freehand $2 \mathrm{~d}$ ultrasound sweeps to $3 \mathrm{~d}$ ct of liver tumours," in Abdominal Imaging Workshop, pp. 155-164. Springer, 2013.

[4] A Yavariabdi, C Samir, et al., "Mapping endometrial implants by $2 \mathrm{~d} / 2 \mathrm{~d}$ registration of tvus to $\mathrm{mr}$ images from point correspondences," in ISBI, 2013.

[5] B Glocker, A Sotiras, N Komodakis, and N Paragios, "Deformable medical image registration: setting the state of the art with discrete methods.," Апnи Rev Biomed Eng, vol. 13, 2011.

[6] E Ferrante and N Paragios, "Non-rigid 2d-3d medical image registration using markov random fields," in MICCAI 2013. 2013, pp. 163-170, Springer.

[7] D Kwon, K Joon Lee, I Dong Yun, and S Lee, "Nonrigid image registration using dynamic higher-order mrf model," in ECCV 2008, pp. 373-386. Springer, 2008.

[8] JH. Kappes, B Andres, et al., "A comparative study of modern inference techniques for discrete energy minimization problem," in CVPR, 2013. 